\title{
NON-SPECIFIC OESOPHAGITIS
}

\author{
BY \\ K. V. LODGE \\ From the Department of Pathology, the University of Manchester
}

(RECEIVED FOR PUBLICATION OCTOBER 23, 1954)

Non-specific oesophagitis is well recognized as a sequel to operations for the relief of cardiospasm and in association w:th hiatus hernia (Belsey, 1953). The frequency with which oesophagitis occurs in general hospital practice is, however, not widely appreciated. This is probably due mainly to the mildness or absence of symptoms in many cases of oesophagitis. The purpose of the present paper is to stress the frequency of the lesion, particularly as a result of confinement to bed, and to suggest the gravity of the possible clinical sequelae if the condition is unrecognized and untreated.

\section{METHOD}

The findings are based on the examination of the oesophagi from 500 unselected hospital necropsies, and on a similar review of the oesophagi from 100 cases of sudden death. Patients in the latter group had been in apparently good health until the onset of their fatal illnesses, which lasted not longer than 24 hours. Some were the victims of industrial or road accidents; others collapsed and died in the street or at work.

From the pathological aspect, whether there was evidence of oesophagitis macroscopically or not, blocks of the oesophagus were examined microscopically.

Clinically the case history of each patient was reviewed to determine the relationship between oesophagitis and a number of factors considered of importance by previous writers.

\section{RESULTS}

In the 500 hospital patients the incidence of oesophagitis was $36 \%$, composed of $31 \%$ showing an acute lesion and $5 \%$ with chronic oesophagitis. Grouping the cases into three arbitrarily decided grades of severity (Lodge, 1955), 20.5\% of the lesions were of the most advanced type with widespread destruction of the oesophageal mucosa and inflammation extending into the muscularis externa. In the " sudden death" series $8 \%$ had oesophagitis, composed of $3 \%$ showing an acute lesion and $5 \%$ chronic oesophagitis with fibrosis of the wall.

An analysis of the clinical features of the hospital cases showed that patients with severe vomiting or in whom gastric intubation was required, as for example in peritonitis, were particularly liable to the lesion.

\section{Discussion}

The incidence of non-specific oesophagitis in $36 \%$ of unselected hospital necropsies greatly ex- O ceeds that of any previous report. Bartels (1935), for example, described an incidence of only $z$ $0.013 \%$, and Butt and Vinson (1936b) found the lesion in only $7.02 \%$ of their series. The present $\stackrel{5}{工}$ investigation, however, is the first in which all $\overrightarrow{0}$ specimens were examined microscopically irrespec- $f$ tive of naked-eye appearances. This may well explain the higher incidence, for not only is the de-O tection of mild oesophagitis difficult or impossible macroscopically, but even the more severe grades may be masked when there is also post-mortem change.

Excessive regurgitation of acid gastric contents $\overrightarrow{\vec{O}}$ into the oesophagus appears the most important factor in producing oesophagitis. Some regurgitation without apparent pathological change may? occur (Robins and Jankelson, 1926 ; Lawler and McCreath, 1951), but the effects of unusually fre-음 quent or prolonged contact between stomach con-x tents and the oesophageal mucosa are illustrated윽 by the particular incidence of oesophagitis in severe vomiting or after prolonged gastric intubation. Intubation introduces the further factor of trauma to the mucosa. This was shown to be of import-o ance by Friedenwald, Feldman, and Zinn (1928), who found that experimentally produced ulcers in the oesophagus of the dog failed to heal in then presence of acid.

Apart from vomiting and intubation, the ${ }^{N}$ recumbent posture of the patient confined to bed also facilitates unusual regurgitation intoo the oesophagus. This postural factor probablye accounts to a very great extent for the incidence of $36 \%$ of oesophagitis in the hospital series of cases. This is emphasized by comparison with the far lower incidence of $8 \%$ in the "sudden death" series in which the patients were ambulatant until at the most 24 hours befores their demise. An additional factor in the bedridden patient is loss of muscle tone, particularly 
in the diaphragm. This produces an impairment in the action of diaphragmatic "pinchcock" on the lower oesophagus. Severe infections and debilitating diseases may also be of importance, in that they may lower the resistance of the tissues of the oesophagus to the harmful effects of minor trauma and regurgitated acid.

There is thus good evidence that quite apart from the specialized surgery of the lower oesophagus, as in the relief of achalasia, general medical or surgical ills which confine the patient to bed lead to oesophagitis in an appreciable number of cases.

The typically described symptoms of oesophagitis are a burning pain deep to the lower end of the sternum, dysphagia, and flatulence. Haematemesis may also occur. The symptoms, however, vary greatly in severity. Many patients, particularly those with only a mild degree of oesophagitis, do not complain of symptoms and the diagnosis is only made at necropsy after death from some other cause.

Evaluation of the symptoms, however, is unusually difficult, for when the patient is suffering from some other disease its symptoms may overshadow those of the oesophagitis. Previous conclusions, such as those of Bartels (1935) and Butt and Vinson (1936a and b), were in retrospect, for they relied on a survey of the clinical notes after the post-mortem findings. This was also the case in the present work, and as all the information required had not been recorded in the case-notes it was not possible to make a full analysis of the clinical features of the patients in the series. However, where possible, the medical staff who had attended these patients were questioned at the time of necropsy to ascertain whether there had been even minor complaints such as flatulence, upper abdominal discomfort, "indigestion," or any other symptoms which might have been dismissed as irrelevant to the main diagnosis. This enquiry confirmed the striking absence in many instances of any symptoms suggestive of oesophagitis even when necropsy revealed a severe lesion. An accurate examination of a large series of patients, bearing the possibility of the disease in mind, correlated with necropsy findings would be both interesting and instructive. It has been recognized for many years that extensive ulceration of the oesophagus may lead to a degree of stenosis severe enough to cause death by starvation (Basham, 1850). Allison, Johnstone, and Royce (1943) and Stephens (1949) have stressed that many of the socalled cases of " congenital short oesophagus" are in fact acquired lesions due to fibrosis following oesophagitis. In the present review $20.5 \%$ of the cases of oesophagitis were severe (Grade 3) and showed widespread destruction of the mucosa and submucosa with inflammation in the muscularis externa. Appreciable scarring with stenosis and shortening of the oesophagus would have been likely in such cases had the patients lived. Such a possibility of serious sequelae justifies preventive measures in all patients confined to bed, and a constant awareness of the symptoms of oesophagitis in these cases so that early treatment may be instituted to prevent progression to the more severe and chronic lesion.

\section{SUMMARY}

Non-specific oesophagitis is discussed with particular reference to its clinical importance.

The study is based on the necropsy findings on 500 general hospital patients and 100 cases of sudden death.

The relative frequency with which oesophagitis occurs in patients confined to bed is shown by its incidence of $36 \%$ in the hospital series of cases, and is contrasted with an incidence of only $8 \%$ in the " sudden death" series.

The importance of reflux of gastric contents in the aetiology of the disease is illustrated by the particular tendency of oesophagitis to occur in patients with severe vomiting or in whom prolonged gastric intubation is required. The effect of the horizontal posture and weakness of the diaphragmatic musculature in producing a similar reflux in bed-ridden patients is stressed.

The frequent clinical silence of oesophagitis is noted. A correlation between clinical and necropsy findings, at present inadequate, is required to facilitate early diagnosis of the disease.

Shortening and stricture of the oesophagus are noted as possible sequelae of the more severe lesions.

It is stressed that oesophagitis is a complication worthy of preventive measures by the general physician and general surgeon.

I wish to thank Professor A. C. P. Campbell for his helpful criticism and advice, and Dr. T. H. Blench and Dr. L. Stent, who provided specimens from cases of sudden death.

\section{REFERENCES}

Allison, P. R., Johnstone, A. S., and Royce, G. B. (1943). J. thorac. Surg., 12, 432.

Bartels, E. C. (1935). Arch. Path., Chicago, 20, 369

Basham, W. (1850). Med.-chir. Trans., 33, 99.

Belsey, R. (1953). Proc. roy. Soc. Med., 46, 941

Butt, H. R., and Vinson, P. P. (1936a). Arch. Otolaryng., Chicago, 23, 391 .

(1936b). Ibid., 23, 550.

Friedenwald, J., Feldman, M., and Zinn, W. F. (1928). Arch. intern. Med., 42, 521.

Lawler, N. A., and McCreath, N. D. (1951). Lancet, 2, 369.

Lodge, K. V. (1955). J. Path. Bact. 69, 17.

Robins, S. A., and Jankelson, I. R. (1926). J. Amer. med. Ass., 87, 1961 Stedhens, H. B. (1949). Calif. Med., 71, 38. 